\title{
The dielectric tensor for dusty magnetized plasmas with variable charge on dust particles
}

\author{
M. C. de JULI† and R. S. SCHNEIDER \\ Instituto de Física, Universidade Federal do Rio Grande do Sul, Caixa Postal 15051, \\ 91501-970 Porto Alegre, RS, Brasil
}

(Received 23 June 1997 and in revised form 5 January 1998)

We derive the dielectric tensor for multicomponent magnetized dusty plasmas, including the effect of capture of plasma electrons and ions by the dust particles. For propagation perpendicular to the external magnetic field and Maxwellian distributions of electrons and ions, we obtain compact expressions for the components of the dielectric tensor, which can be used to analyse wave propagation. An application to the magnetosonic wave is presented.

\section{Introduction}

In recent years, interest in dusty plasmas has increased significantly because it has been recognized that they are important for a number of applications in space plasmas and in the Earth's environment, as well as in laboratory plasmas and in several technologies (D'Angelis 1992).

A dusty plasma may be defined as an ensemble of dust particles immersed in a fully ionized plasma composed of electrons and ions. The dust particles may possess variable mass, size and shape, and, predominantly, fluctuating charge. In general, dust particles are highly charged (of order $10^{3} e-10^{4} e$ ) with variable sizes $(10 \mathrm{~nm}-$ $100 \mu \mathrm{m})$ and masses. The assumption that the dust particles are spherical point masses with constant radius can be made as a first approximation, but the charge acquired by them must be taken into account. The dust particles in a dusty plasma can be charged by plasma current, photoemission, secondary emission, etc. (Draine and Salpeter 1979).

Several analyses, treating the dust as a charged-particle species of uniform mass and charge, have shown that the presence of the charged dust component leads to the appearance of new plasma modes arising from the dust-particle dynamics or to modification of the existing plasma modes (Rao et al. 1990; Shukla 1992; Rao 1995). In recent years, several works that have taken account of the charge variation of the dust particles (Melandso et al. 1993; Varma et al. 1993; Vladimirov 1994) have pointed out the importance of this feature as a source of damping or amplification of waves propagating in dusty plasmas. These analyses describe the dusty plasma in the framework of kinetic theory, but, in general, they concentrate on obtaining the dielectric tensor for a particular mode of propagation, except in the work of Vladimirov (1994), where general expressions for the dielectric tensor are obtained, but in the absence of an external magnetic field. To the best of our knowledge,

$\dagger$ E-mail: juli@if.ufrgs.br. 
general expressions for the components of the dielectric tensor of a dusty plasma with variable charge of the dust particles, in the presence of an external magnetic field, have not previously been obtained.

In this work we develop a kinetic theory of dusty magnetized plasmas with variable charges on the dust particles. The dust particles are assumed to be immobile, and consequently the validity of the proposed model will be restricted to waves with frequency much higher than the characteristic dust frequencies. We observe that the dominant role of the dust particles in the process of wave propagation is due to charge variation and that this modelling excludes the possibility of analysing modes that can arise from the dust dynamics. Exact expressions for the components of the dielectric tensor are derived and then particularized to propagation perpendicular to the external magnetic field, and Maxwellian distributions of electrons and ions. The expressions obtained show that there are two kinds of modifications introduced by the presence of dust particles with variable charges. The dielectric tensor can be written as the sum of two terms. One term is formally identical to the dielectric tensor of a homogeneous magnetized plasma, with the resonant denominator changed by the addition of a purely imaginary term containing a frequency that characterizes the collisions between plasma particles and dust particles. The second term is entirely dependent on the model used to describe the process of charging of the dust particles.

We intend to apply this dielectric tensor to analyse several modes of propagation, trying to understand the importance of the charge variation as a mechanism of damping or amplification. We shall also try to analyse the competition that may arise between Landau damping and the damping due to the presence of dust particles with variable charges.

The plan of the paper is as follows. In Sec. 2 we present the basic equations of the model proposed for the dusty plasma, linearize these equations and analyse the quantities that characterize the equilibrium of the system. In Sec. 3 the derivation of the dielectric tensor is presented, restricted only by the requirements imposed by equilibrium. In Sec. 4 complete expressions for the components of the dielectric tensor are presented for propagation perpendicular to the external magnetic field and for Maxwellian distribution functions of electrons and ions. In Sec. 5 a preliminary application of the developed formalism is made to the magnetosonic wave, showing that the charge variation of the dust particles in a plasma gives the possibility of absorption. Some numerical results that show the dependence of the damping rate on temperature and on the external magnetic field have been included. Finally, in Sec. 6 we summarize our results and drawn some conclusions.

\section{The basic equations of the model}

We consider a homogeneous plasma composed of particles of charge $q_{\beta}$ and mass $m_{\beta}$, where the subscript $\beta=e, i$ identifies electrons and ions respectively, in a homogeneous external magnetic field $\mathbf{B}_{0}=B_{0} \mathbf{e}_{z}$. In this magnetized plasma we consider embedded spherical dust grains with radius $a$ and variable charge $q$; this variation originates from collisions between the dust particles and particles of species $\beta$. The assumption of spherical dust grains is valid in the limit $a \ll \lambda_{D} \ll \lambda_{m f p}$, where $\lambda_{D}$ is the plasma Debye length and $\lambda_{m f p}$ is the mean free path of the electrons and ions. We observe that most astrophysical and laboratory dusty plasmas satisfy this condition. The charging model for the dust particles must take into account the 
presence of an external magnetic field. This field must influence the characteristics of charging of the dust particles, because the path described by electrons and ions is drastically changed: in this case we have cyclotron motion of electrons and ions around the magnetic field lines. It has been shown (Hutchinson 1987; Chang and Spariosu 1993) that for $a \ll \rho$, where $\rho$ is the electron gyroradius, the effect of the magnetic field on the charging of the dust particles can be neglected.

The distribution function that describes the dust particles, which are assumed to be immobile because of their large mass, is $f_{d} \equiv f_{d}(q, \mathbf{r}, t)$ and is the solution of the equation

$$
\frac{\partial f_{d}}{\partial t}+\frac{\partial}{\partial q}\left[I(q) f_{d}\right]=0
$$

where

$$
I(q)=\sum_{\beta} \int d^{3} p q_{\beta} \sigma_{\beta}(q, p) \frac{p}{m_{\beta}} f_{\beta}
$$

is the current on the dust particle (Tsytovich and Havnes 1993), $f_{\beta}(\mathbf{r}, \mathbf{p}, t)$ the distribution function of particles of species $\beta$, and $\sigma_{\beta}$ is the charging cross-section, given (Spitzer 1978) by

$$
\sigma_{\beta}=\pi a^{2}\left(1-\frac{2 q q_{\beta} m_{\beta}}{a p^{2}}\right) H\left(1-\frac{2 q q_{\beta} m_{\beta}}{a p^{2}}\right),
$$

where $H$ denotes the Heaviside function.

The distribution function of electrons and ions satisfies a Vlasov equation with a collision term, which assures the possibility of variation of charge of the dust particles:

$$
\begin{array}{r}
\frac{\partial f_{\beta}}{\partial t}+\frac{1}{m_{\beta} \gamma_{\beta}} \mathbf{p} \cdot \nabla \\
f_{\beta}+q_{\beta}\left(\mathbf{E}+\frac{1}{m_{\beta} \gamma_{\beta} c} \mathbf{p} \times \mathbf{B}\right) \cdot \nabla_{\mathbf{p}} f_{\beta} \\
=-\int \sigma_{\beta} \frac{p}{m_{\beta} \gamma_{\beta}}\left(f_{d} f_{\beta}-f_{d 0} f_{\beta 0}\right) d q,
\end{array}
$$

where $\gamma_{\beta}=\left(1+p^{2} / m_{\beta}^{2} c^{2}\right)^{1 / 2}$ is the relativistic factor.

The connection with the fields is described by the Maxwell equations:

$$
\begin{gathered}
\nabla \cdot \mathbf{E}=4 \pi\left(\sum_{\beta} q_{\beta} \int f_{\beta} d^{3} p+\int q f_{d} d q\right), \\
\nabla \times \mathbf{E}=-\frac{1}{c} \frac{\partial \mathbf{B}}{\partial t}, \\
\nabla \cdot \mathbf{B}=0, \\
\nabla \times \mathbf{B}=\frac{1}{c} \frac{\partial \mathbf{E}}{\partial t}+\frac{4 \pi}{c} \sum_{\beta} \frac{q_{\beta}}{m_{\beta}} \int \frac{\mathbf{p}}{\gamma_{\beta}} f_{\beta} d^{3} p .
\end{gathered}
$$

The set of equations (2.1)-(2.7) is linearized, resulting in the following zerothorder equations, where we assume $f_{\beta 0} \equiv f_{\beta 0}(\mathbf{p})$ and $f_{d 0} \equiv f_{d 0}(q)$ :

$$
\frac{\partial}{\partial q}\left[I_{0}(q) f_{d 0}\right]=0
$$




$$
\begin{gathered}
I_{0}(q)=\sum_{\beta} \int d^{3} p q_{\beta} \sigma_{\beta}(q, p) \frac{p}{m_{\beta}} f_{\beta 0}, \\
\left(\mathbf{p} \times \mathbf{B}_{0}\right) \cdot \nabla_{\mathbf{p}} f_{\beta 0}=0 \\
\sum_{\beta} q_{\beta} \int f_{\beta 0} d^{3} p+\int q f_{d 0} d q=0 \\
\sum_{\beta} \frac{q_{\beta}}{m_{\beta}} \int \frac{\mathbf{p}}{\gamma_{\beta}} f_{\beta 0} d^{3} p=0 .
\end{gathered}
$$

We also define the auxiliary function $\nu_{\beta d}^{0}(p)$ :

$$
\nu_{\beta d}^{0}(p) \equiv \int \sigma_{\beta} \frac{p}{m_{\beta}} f_{d 0} d q .
$$

These equations that describe the equilibrium are satisfied if $f_{\beta 0}$ is a function with azimuthal symmetry: $f_{\beta 0}=f_{\beta 0}\left(p_{\perp}, p_{\|}\right)$. Using $f_{d 0}=n_{d 0} \delta\left(q+Z_{d} e\right)$, where $-Z_{d} e$ is the equilibrium charge of the dust particles, we obtain

$$
\nu_{\beta d}^{0}(p)=\frac{n_{d 0} p}{m_{\beta}} \sigma_{\beta}\left(p,-Z_{d} e\right)=\frac{\pi a^{2} n_{d 0}}{m_{\beta}} \frac{\left(p^{2}+C_{\beta}\right)}{p} H\left(p^{2}+C_{\beta}\right),
$$

where $C_{\beta} \equiv 2 Z_{d} e q_{\beta} m_{\beta} / a$. Also, the quasineutrality condition is given by

$$
\sum_{\beta} q_{\beta} n_{\beta 0}=Z_{d} e n_{d 0}
$$

The auxiliary function $\nu_{\beta d}^{0}(p)$ is related to the frequency characterizing the rate of capture of particles of species $\beta$ by dust particles in the equilibrium state, which can be obtained from

$$
\nu_{\beta d}=\frac{1}{n_{\beta 0}} \int d^{3} p \nu_{\beta d}^{0}(p) f_{\beta 0} .
$$

Using the expression for $\sigma_{\beta}$, the current on the dust particles in equilibrium can be written as

$$
I_{0}(q)=\pi a^{2} \sum_{\beta} \frac{q_{\beta}}{m_{\beta}} \int d^{3} p \frac{1}{p}\left(p^{2}-C_{\beta} \frac{q}{Z_{d} e}\right) H\left(p^{2}-C_{\beta} \frac{q}{Z_{d} e}\right) f_{\beta 0} .
$$

In this calculation we have assumed that $q<0$, that is, the net charge acquired by the dust particles is negative. For a Maxwellian distribution of particles of species $\beta$,

$$
f_{\beta 0}=\frac{n_{\beta 0}}{\left(2 \pi m_{\beta} T_{\beta}\right)^{3 / 2}} e^{-p^{2} / 2 m_{\beta} T_{\beta}},
$$

we obtain

$$
I_{0}(q)=2 a^{2}(2 \pi)^{1 / 2} \sum_{\beta} q_{\beta} n_{\beta 0}\left(\frac{T_{\beta}}{m_{\beta}}\right)^{1 / 2}\left(1-\frac{e q}{T_{e} a} \delta_{\beta e}-\frac{q_{\beta} q}{T_{\beta} a}\right) e^{e q \delta_{\beta e} / T_{e} a},
$$

where $\delta_{\beta e}$ is the Kronecker delta symbol.

The equilibrium charge of the dust particles, $-Z_{d} e$, is obtained by imposing the condition that

$$
I_{0}\left(-Z_{d} e\right)=0
$$


From $(2.18)$ we get a relation from which we can determine $Z_{d}$ :

$$
\frac{\omega_{p e}^{2}}{v_{T e}} e^{-\left|\chi_{e}\right|}=\tau \frac{\omega_{p i}^{2}}{v_{T i}}\left(1+\frac{\left|\chi_{e}\right|}{\tau}\right),
$$

where

$$
\begin{gathered}
v_{T \beta} \equiv\left(\frac{T_{\beta}}{m_{\beta}}\right)^{1 / 2}, \\
\tau=\frac{e}{q_{i}} \frac{T_{i}}{T_{e}}=-\frac{\chi_{e}}{\chi_{i}}, \\
\chi_{\beta}=\frac{Z_{d} q_{\beta} e}{T_{\beta} a} .
\end{gathered}
$$

The first-order equations arising from the set of equations (2.1)-(2.7) are

$$
\begin{gathered}
\frac{\partial f_{d 1}}{\partial t}+\frac{\partial}{\partial q}\left[I_{0}(q) f_{d 1}+I_{1}(q) f_{d 0}\right]=0 \\
I_{1}(q) \equiv \sum_{\beta} \int d^{3} p \frac{q_{\beta}}{m_{\beta}} \sigma_{\beta}(q, p) p f_{\beta 1}, \\
\nu_{\beta d}^{1}(p) \equiv \int \sigma_{\beta}(q, p) \frac{p}{m_{\beta}} f_{d 1} d q, \\
\frac{\partial f_{\beta 1}}{\partial t}+\frac{1}{m_{\beta} \gamma_{\beta}} \mathbf{p} \cdot \nabla f_{\beta 1}+\frac{q_{\beta}}{m_{\beta} \gamma_{\beta} c}\left(\mathbf{p} \times \mathbf{B}_{0}\right) \cdot \nabla_{\mathbf{p}} f_{\beta 1}+\nu_{\beta d}^{0}(p) f_{\beta 1} \\
=-\nu_{\beta d}^{1}(p) f_{\beta 0}-q_{\beta}\left(\mathbf{E}_{1}+\frac{1}{m_{\beta} \gamma_{\beta} c} \mathbf{p} \times \mathbf{B}_{1}\right) \cdot \nabla_{\mathbf{p}} f_{\beta 0}, \\
\nabla \times \mathbf{E}_{1}=4 \pi\left(\sum_{\beta} q_{\beta} \int f_{\beta 1} d^{3} p+\int q f_{d 1} d q\right) \\
\nabla \times \mathbf{E}_{1}=-\frac{1}{c} \frac{\partial \mathbf{B}_{1}}{\partial t}, \\
\frac{\nabla \mathbf{E}_{1}}{\partial t}+\frac{4 \pi}{c} \sum_{\beta} \frac{q_{\beta}}{m_{\beta}} \int \frac{\mathbf{p}}{\gamma_{\beta}} f_{\beta 1} d^{3} p .
\end{gathered}
$$

We note that if we exclude the charge variation of the dust particles from the proposed model, this will be equivalent to taking $\sigma(q, p)=0$. Thus our model will retain information about the presence of the dust particles in the plasma only in the quasineutrality condition. The dielectric tensor that we obtain in this case is the same as that obtained for a magnetized plasma of electrons and ions, but the value of some components of this tensor will be modified because the quasineutrality condition has changed. When we discuss the application to the magnetosonic wave, we shall return to this point. 


\section{Derivation of the dielectric tensor}

From the linearized system of equations $(2.24)-(2.31)$, we shall obtain the dielectric tensor for the dusty plasma considered. We note that this linearized system is a coupled system of integro-differential equations, and in order to get the algebraic system of equations that leads to the dispersion tensor, some approximations must be made.

We take some moments of the distribution function of the dust particles, $f_{d}(q, \mathbf{r}, t)$, in the variable $q$. The zeroth-order moment is given by

$$
\int f_{d}(q, \mathbf{r}, t) d q=n_{d}(\mathbf{r}, t)
$$

where the integration is from $-\infty$ to 0 , because we have assumed in the calculation of $I_{0}(q)$ that $q<0$. Linearizing this relation, we get, at zeroth order,

$$
\int f_{d 0} d q=n_{d 0},
$$

which is satisfied if $f_{d 0}=n_{d 0} \delta\left(q+Z_{d} e\right)$, and, at first order,

$$
\int f_{d 1} d q=n_{d 1}(\mathbf{r}, t)
$$

The first-order moment in the variable $q$ is

$$
\int f_{d}(q, \mathbf{r}, t) q d q=n_{d 0} Q(\mathbf{r}, t),
$$

which, after linearization, gives, at zero order, $Q_{0}=-Z_{d} e$, and, at first order,

$$
\int f_{d \mathbf{1}} q d q=n_{d 0} Q_{1}(\mathbf{r}, t)
$$

The linearized system and (3.3) and (3.5) are Fourier-transformed in space and time:

$$
A_{1}(\Gamma, \mathbf{r}, t) \longrightarrow \hat{A}(\Gamma, \mathbf{k}, \omega) e^{i(\mathbf{k} \cdot \mathbf{r}-\omega t)},
$$

where $A_{1}$ denotes any of the perturbed variables and $\Gamma$ represents $\mathbf{p}$ or $q$. We obtain

$$
\begin{gathered}
-i \omega \hat{f}_{d}+\frac{\partial}{\partial q}\left[I_{0}(q) \hat{f}_{d}+\hat{I}(q) f_{d 0}\right]=0 \\
\hat{I}(q) \equiv \sum_{\beta} \int d^{3} p \frac{q_{\beta}}{m_{\beta}} \sigma_{\beta}(q, p) p \hat{f}_{\beta} \\
-i \omega \hat{f}_{\beta}+\frac{i}{m_{\beta} \gamma_{\beta}} \mathbf{k} \cdot \mathbf{p} \hat{f}_{\beta}+\frac{q_{\beta}}{m_{\beta} \gamma_{\beta} c}\left(\mathbf{p} \times \mathbf{B}_{0}\right) \cdot \nabla_{\mathbf{p}} \hat{f}_{\beta}+\nu_{\beta d}^{0}(p) \hat{f}_{\beta} \\
=-\hat{\nu}_{\beta d}(p) f_{\beta 0}-q_{\beta}\left(\hat{\mathbf{E}}+\frac{1}{m_{\beta} \gamma_{\beta} c} \mathbf{p} \times \hat{\mathbf{B}}\right) \cdot \nabla_{\mathbf{p}} f_{\beta 0} \\
\hat{\nu}_{\beta d}(p)=\int \sigma_{\beta}(q, p) \frac{p}{m_{\beta}} \hat{f}_{d} d q \\
i \mathbf{k} \cdot \hat{\mathbf{E}}=4 \pi\left(\sum_{\beta} q_{\beta} \int \hat{f}_{\beta} d^{3} p+\int q \hat{f}_{d} d q\right)
\end{gathered}
$$




$$
\begin{aligned}
\mathbf{k} \times \hat{\mathbf{E}} & =\frac{\omega}{c} \hat{\mathbf{B}}, \\
\mathbf{k} \cdot \hat{\mathbf{B}} & =0,
\end{aligned}
$$

$$
i \mathbf{k} \times \hat{\mathbf{B}}=-i \frac{\omega}{c} \hat{\mathbf{E}}+\frac{4 \pi}{c} \sum_{\beta} \frac{q_{\beta}}{m_{\beta}} \int \frac{\mathbf{p}}{\gamma_{\beta}} \hat{f}_{\beta} d^{3} p
$$

$$
\begin{gathered}
\int \hat{f}_{d} d q=\hat{n}_{d}, \\
\int \hat{f}_{d} q d q=n_{d 0} \hat{Q}
\end{gathered}
$$

We rewrite $(2.27)$ in the form

$$
\frac{\partial f_{\beta}^{\text {aux }}}{\partial t}+\frac{1}{m_{\beta} \gamma_{\beta}} \mathbf{p} \cdot \nabla f_{\beta}^{\text {aux }}+\frac{q_{\beta}}{m_{\beta} \gamma_{\beta} c}\left(\mathbf{p} \times \mathbf{B}_{0}\right) \cdot \nabla_{\mathbf{p}} f_{\beta}^{\text {aux }}=-e^{\nu_{\beta d}^{0}(p) t}(O p) f_{\beta 0},
$$

where

$$
\begin{gathered}
(O p) \equiv \nu_{\beta d}^{1}(p)+q_{\beta}\left(\mathbf{E}_{1}+\frac{1}{m_{\beta} \gamma_{\beta} c} \mathbf{p} \times \mathbf{B}_{1}\right) \cdot \nabla_{\mathbf{p}}, \\
f_{\beta 1}=e^{-\nu_{\beta d}^{0}(p) t} f_{\beta}^{\text {aux }} .
\end{gathered}
$$

Integrating 3.17 over the unperturbed orbits we obtain

$$
\begin{aligned}
f_{\beta \mathbf{1}}(\mathbf{p}, \mathbf{r}, t)= & -e^{-\nu_{\beta d}^{0}(p) t} \int_{-\infty}^{t} d t^{\prime} e^{\nu_{\beta d}^{0}\left(p^{\prime}\right) t^{\prime}}\left\{\nu_{\beta d}^{1}\left(p^{\prime}, \mathbf{r}^{\prime}, t^{\prime}\right)\right. \\
& \left.+q_{\beta}\left[\mathbf{E}_{1}\left(\mathbf{r}^{\prime}, t^{\prime}\right)+\frac{1}{m_{\beta} \gamma_{\beta} c} \mathbf{p}^{\prime} \times \mathbf{B}_{1}\left(\mathbf{r}^{\prime}, t^{\prime}\right)\right] \cdot \nabla_{\mathbf{p}^{\prime}}\right\} f_{\beta 0}\left(\mathbf{p}^{\prime}\right),
\end{aligned}
$$

where the unperturbed orbits are given by (Krall and Trivelpiece 1973)

$$
\begin{gathered}
x^{\prime}-x=-\frac{p_{\perp}}{m_{\beta} \Omega_{\beta}}\left[\sin \left(\varphi-\frac{\Omega_{\beta} \tau}{\gamma_{\beta}}\right)-\sin \varphi\right], \\
y^{\prime}-y=\frac{p_{\perp}}{m_{\beta} \Omega_{\beta}}\left[\cos \left(\varphi-\frac{\Omega_{\beta} \tau}{\gamma_{\beta}}\right)-\cos \varphi\right], \\
z^{\prime}-z=\frac{p_{\|}}{m_{\beta} \gamma_{\beta}} \tau, \\
p_{x}^{\prime}=p_{\perp} \cos \left(\varphi-\frac{\Omega_{\beta} \tau}{\gamma_{\beta}}\right), \\
p_{y}^{\prime}=p_{\perp} \sin \left(\varphi-\frac{\Omega_{\beta} \tau}{\gamma_{\beta}}\right), \\
p_{z}^{\prime}=p_{\|},
\end{gathered}
$$

where $\tau=t^{\prime}-t$, and $\Omega_{\beta}=q_{\beta} B_{0} / m_{\beta} c$ is the gyrofrequency of particles of species $\beta$.

The equilibrium distribution function $f_{\beta 0}$ is an arbitrary function of the constants of motion, which in this case are $p_{\perp}$ and $p_{\|}$, as can easily be obtained from the equations for the unperturbed orbits. The Fourier-Laplace transform of (3.20) can 
be written as

$$
\begin{aligned}
\hat{f}_{\beta}(\mathbf{p})=-\int_{-\infty}^{0} d \tau e^{\nu_{\beta d}^{0}(p) \tau} e^{i(\mathbf{k} \cdot \mathbf{R}-\omega \tau)}\left[\hat{\nu}_{\beta d}(p)\right. & \\
& \left.+q_{\beta}\left(\hat{\mathbf{E}}+\frac{1}{m_{\beta} \gamma_{\beta} c} \mathbf{p}^{\prime} \times \hat{\mathbf{B}}\right) \cdot \nabla_{\mathbf{p}^{\prime}}\right] f_{\beta 0}\left(p_{\perp}, p_{\|}\right),
\end{aligned}
$$

where $\mathbf{R}=\mathbf{r}^{\prime}-\mathbf{r}$.

In order to clarify the derivation that follows, we write the Fourier-Laplace transform of the perturbed distribution function as

$$
\hat{f}_{\beta}(\mathbf{p})=\hat{f}_{\beta}^{h}+\hat{f}_{\beta}^{N}
$$

where

$$
\begin{gathered}
\hat{f}_{\beta}^{h}=-q_{\beta} \int_{-\infty}^{0} d \tau e^{i\left\{\mathbf{k} \cdot \mathbf{R}-\left[\omega+i \nu_{\beta d}^{0}(p)\right] \tau\right\}}\left(\hat{\mathbf{E}}+\frac{1}{m_{\beta} \gamma_{\beta} c} \mathbf{p}^{\prime} \times \hat{\mathbf{B}}\right) \cdot \nabla_{\mathbf{p}^{\prime}} f_{\beta 0}\left(p_{\perp}, p_{\|}\right), \\
\hat{f}_{\beta}^{N}=-\int_{-\infty}^{0} d \tau e^{i\left[\mathbf{k} \cdot \mathbf{R}-\left(\omega+i \nu_{\beta d}^{0}\right) \tau\right]} \hat{\nu}_{\beta d}(p) f_{\beta 0} .
\end{gathered}
$$

The components of the dielectric tensor can be written as

$$
\epsilon_{i j}=\tilde{\epsilon}_{i j}^{h}+\epsilon_{i j}^{N}
$$

where the first term originates from $\hat{f}_{\beta}^{h}$ and the second from $\hat{f}_{\beta}^{N}$.

We note that $\hat{f}_{\beta}^{h}$ has the same formal structure as the perturbed distribution function obtained when we are calculating the traditional dielectric tensor for a homogeneous magnetized plasma (Caldela et al. 1989), substituting $\omega+i \nu_{\beta d}^{0}(p)$ for $\omega$ in the argument of the exponential. We conclude that this part of the perturbed distribution will give rise to a contribution to the dielectric tensor (whose components we are representing by $\tilde{\epsilon}_{i j}^{h}$ ), that is formally identical to the corresponding components of the dielectric tensor of a homogeneous plasma composed of particles of species $\beta$ in the presence of a external magnetic field, except for the addition in the resonant denominator of a term that takes care of the damping due to collisions between dust particles and the electrons and ions. These components of the dielectric tensor can be written as

$$
\begin{aligned}
\tilde{\epsilon}_{i j}^{h}=\delta_{i j}+\sum_{\beta} \frac{X_{\beta}}{n_{\beta 0}} \sum_{n=-\infty}^{\infty} \int d^{3} p p_{\perp} & \frac{\phi_{0}\left(f_{\beta 0}\right)}{D_{n \beta}}\left(\frac{p_{\|}}{p_{\perp}}\right)^{\delta_{i z}+\delta_{j z}} R_{i j} \\
& -\delta_{i z} \delta_{j z} \sum_{\beta} \frac{X_{\beta}}{n_{\beta 0}} \int d^{3} p \frac{L\left(f_{\beta 0}\right)}{\gamma_{\beta}} \frac{p_{\|}}{p_{\perp}},
\end{aligned}
$$

where

$$
\begin{gathered}
L\left(f_{\beta 0}\right)=p_{\|} \frac{\partial f_{\beta 0}}{\partial p_{\perp}}-p_{\perp} \frac{\partial f_{\beta 0}}{\partial p_{\|}}, \\
\phi_{0}\left(f_{\beta 0}\right)=\frac{\partial f_{\beta 0}}{\partial p_{\perp}}-\frac{k_{\|}}{m_{\beta} \gamma_{\beta} \omega} L\left(f_{\beta 0}\right), \\
D_{n \beta}=\frac{\gamma_{\beta}}{\omega}\left[\omega+i \nu_{\beta d}^{0}(p)-\frac{k_{\|} p_{\|}}{m_{\beta} \gamma_{\beta}}-\frac{n \Omega_{\beta}}{\gamma_{\beta}}\right],
\end{gathered}
$$


the explicit expressions for the $R_{i j}$ are given in Appendix A, and $X_{\beta}=\omega_{p \beta}^{2} / \omega^{2}$, where $\omega_{p \beta}$ is the plasma frequency for particles of species $\beta$.

The second contribution to the dielectric tensor (whose components we are representing by $\epsilon_{i j}^{N}$ ), also comes from the process of charge variation of the dust particles. To derive this contribution, we write, as usual,

$$
\hat{J}_{i}=\sum_{\beta} \frac{q_{\beta}}{m_{\beta}} \int \frac{p_{i}}{\gamma_{\beta}} \hat{f}_{\beta} d^{3} p=\sum_{j} \sigma_{i j} \hat{E}_{j},
$$

which gives

$$
\epsilon_{i j}^{N}=\frac{4 \pi i}{\omega} \sigma_{i j}^{N},
$$

where $\sigma_{i j}^{N}$ must be obtained from the relation

$$
\sum_{j} \sigma_{i j}^{N} \hat{E}_{j}=\sum_{\beta} \frac{q_{\beta}}{m_{\beta}} \int d^{3} p \frac{p_{i}}{\gamma_{\beta}} \hat{f}_{\beta}^{N} .
$$

We see that in order to obtain this contribution to the conductivity tensor, we must express $\hat{f}_{\beta}^{N}$ in terms of the components of the perturbed electric field. Remembering that $p$ is a constant of motion, we can write

$$
\hat{f}_{\beta}^{N}=-I_{\beta}(\mathbf{p}) \hat{\nu}_{\beta d}(p) f_{\beta 0},
$$

where

$$
I_{\beta}(\mathbf{p}) \equiv \int_{-\infty}^{0} d \tau e^{i\left[\mathbf{k} \cdot \mathbf{R}-\left(\omega+i \nu_{\beta d}^{0}\right) \tau\right]},
$$

which can easily be calculated using the unperturbed orbits and (2.14) for $\nu_{\beta d}^{0}$, and $\hat{\nu}_{\beta d}(p)$ is given by (3.10). We see from this equation that $\hat{\nu}_{\beta d}$ depends in an integral form on the complete perturbed distribution $\hat{f}_{d}$. In order to determine this function, we use (3.7), which depends on $\hat{I}(q)$, which in turn depends on an integral form of $\hat{f}_{\beta}$ (see $(3.8)$ ) that is given by (3.28). In other words, the equation that determines $\hat{f}_{d}$ is a complicated integro-differential equation. It is reasonable, for wave frequencies much higher than the characteristic frequencies of the dust particles, to assume that the $q$ dependence of $f_{d}$ follows the same law as $f_{d 0}$ :

$$
\hat{f}_{d}=n_{d 0}\left[\delta(q+x)-\delta\left(q+Z_{d} e\right)\right],
$$

where $x=x(\mathbf{k}, \omega)$ will be determined from the moments of $\hat{f}_{d}$ in the variable $q$. From (3.15), we conclude that $x>0$, and from (3.16) that $x=Z_{d} e-\hat{Q}$. In order to determine $\hat{Q}$, we take the first moment in the $q$ variable of (3.7), getting

$$
n_{d 0} \hat{Q}=\frac{i}{\omega} \int\left[I_{0}(q) \hat{f}_{d}+\hat{I}(q) f_{d 0}\right] d q,
$$

which can be written as

$$
\hat{Q}=\frac{i}{\omega n_{d 0}}\left(T_{1}+T_{2}\right)
$$

where

$$
\begin{aligned}
& T_{1} \equiv \int I_{0}(q) \hat{f}_{d} d q \\
& T_{2} \equiv \int \hat{I}(q) f_{d 0} d q .
\end{aligned}
$$


Using the assumed form for $\hat{f}_{d}$, we get

$$
T_{1}=n_{d 0} I_{0}\left(q=Q_{0}+\hat{Q}\right),
$$

which, if $|\hat{Q}| \ll\left|Q_{0}\right|$, using a Taylor expansion around $Q_{0}=-Z_{d} e$, and retaining only the dominant contribution, can be written as

$$
T_{1} \simeq-n_{d 0} \nu_{d}^{0} \hat{Q}
$$

where

$$
\nu_{d}^{0}=-\left.\frac{\partial I_{0}}{\partial q}\right|_{q=-Z_{d} e} .
$$

Substituting this result into the expression for $\hat{Q}$ and calculating $T_{2}$, we get

$$
\left(1+i \frac{\nu_{d}^{0}}{\omega}\right) \hat{Q}=\frac{i}{\omega} \hat{I}\left(q=-Z_{d} e\right) .
$$

Using (3.8) and (3.28) and remembering (2.14),

$$
\left(1+i \frac{\nu_{d}^{0}}{\omega}\right) \hat{Q}=\frac{i}{\omega n_{d 0}} \sum_{\beta} q_{\beta} \int d^{3} p \nu_{\beta d}^{0}(p)\left(\hat{f}_{\beta}^{h}+\hat{f}_{\beta}^{N}\right) .
$$

Remembering that $\hat{f}_{\beta}^{N}=-I_{\beta}(\mathbf{p}) \hat{\nu}_{\beta d}(p) f_{\beta 0}$, we calculate $\hat{\nu}_{\beta d}(p)$ using the assumed form for $\hat{f}_{d}$ and making a Taylor expansion of $\sigma_{\beta}$ around $q=-Z_{d} e$, retaining only the relevant term. We obtain

$$
\hat{\nu}_{\beta d}(p) \simeq \frac{n_{d 0}}{m_{\beta}} p \sigma_{\beta}^{\prime}(p) \hat{Q}
$$

where

After some algebra, we can write

$$
\left.\sigma_{\beta}^{\prime}(p) \equiv \frac{\partial \sigma_{\beta}}{\partial q}\right|_{q=-Z_{d} e} .
$$

$$
\hat{Q}=\frac{i}{n_{d 0}\left[\omega+i\left(\nu_{d}^{0}+\nu_{1}\right)\right]} \sum_{\beta} q_{\beta} \int d^{3} p \nu_{\beta d}^{0}(p) \hat{f}_{\beta}^{h}
$$

where

$$
\nu_{1} \equiv \sum_{\beta} \frac{q_{\beta}}{m_{\beta}} \int d^{3} p \nu_{\beta d}^{0}(p) I_{\beta}(\mathbf{p}) p \sigma_{\beta}^{\prime}(p) f_{\beta 0} .
$$

Using these results, we can finally write

$$
\begin{gathered}
\hat{f}_{\beta}^{N}=-\frac{i}{m_{\beta}\left[\omega+i\left(\nu_{d}^{0}+\nu_{1}\right)\right]} I_{\beta}(\mathbf{p}) p \sigma_{\beta}^{\prime}(p) f_{\beta 0} \sum_{\alpha} q_{\alpha} \int d^{3} p \nu_{\alpha d}^{0}(p) \hat{f}_{\alpha}^{h}, \\
\epsilon_{i j}^{N}=-\frac{4 \pi i n_{d 0}}{\omega} U_{i} S_{j},
\end{gathered}
$$

where

$$
\begin{gathered}
U_{i}=\frac{\omega}{\omega+i\left(\nu_{d}^{0}+\nu_{1}\right)} \sum_{\beta} \frac{q_{\beta}}{m_{\beta}^{2}} \int d^{3} p \frac{p_{i}}{\gamma_{\beta}} I_{\beta}(\mathbf{p}) p \sigma_{\beta}^{\prime}(p) f_{\beta 0}, \\
S_{j}=-\frac{i}{\omega n_{d 0}} \sum_{\beta} q_{\beta}^{2} \int d^{3} p \nu_{\beta d}^{0}(p) A_{\beta j},
\end{gathered}
$$


with

$$
A_{\beta j} \equiv \int_{-\infty}^{0}\left[\left(1-\frac{\mathbf{p}^{\prime} \cdot \mathbf{k}}{m_{\beta} \gamma_{\beta} \omega}\right) \frac{\partial}{\partial p_{j}^{\prime}} f_{\beta 0}+\frac{1}{m_{\beta} \gamma_{\beta} \omega}\left(\mathbf{k} \cdot \nabla_{\mathbf{p}^{\prime}} f_{\beta 0}\right) p_{j}^{\prime}\right] e^{i\left\{\mathbf{k} \cdot \mathbf{R}-\left[\omega+i \nu_{\beta d}^{0}(p)\right] \tau\right\}} d \tau .
$$

\section{The dielectric tensor for $k_{\|}=0$ and Maxwellian distributions of electrons and ions}

We shall particularize the general expressions for the dielectric tensor obtained in Sec. 3. We consider propagation perpendicular to the external magnetic field, choosing the coordinate axes in such a way that $\mathbf{k}=k \mathbf{e}_{x}$. We also assume distribution functions to describe electrons and ions in equilibrium such that $f_{\beta 0} \equiv f_{\beta 0}(p)$ and consider the nonrelativistic approximation $\gamma_{\beta}=1$. It is easy to show that the dielectric tensor reduces, without any approximations, to the form

$$
\boldsymbol{\epsilon}=\left(\begin{array}{ccc}
\tilde{\epsilon}_{11}^{h}+\epsilon_{11}^{N} & \tilde{\epsilon}_{12}^{h}+\epsilon_{12}^{N} & 0 \\
-\tilde{\epsilon}_{12}^{h}+\epsilon_{21}^{N} & \tilde{\epsilon}_{22}^{h}+\epsilon_{22}^{N} & 0 \\
0 & 0 & \tilde{\epsilon}_{33}^{h}
\end{array}\right),
$$

where the non-null components that occur in the above expression are given by

$$
\tilde{\epsilon}_{i j}^{h}=\delta_{i j}+\sum_{\beta} \frac{X_{\beta}}{n_{\beta 0}} \sum_{n=-\infty}^{\infty} \int d^{3} p p_{\perp} \frac{\partial f_{\beta 0}}{\partial p_{\perp}} \frac{\Pi_{i j}^{n}}{D_{n \beta}}
$$

with

$$
\begin{gathered}
\Pi_{11}^{n}=\frac{n^{2}}{b_{\beta}^{2}} J_{n}^{2}\left(b_{\beta}\right), \quad \Pi_{12}^{n}=i \frac{n}{b_{\beta}} J_{n}\left(b_{\beta}\right) J_{n}^{\prime}\left(b_{\beta}\right), \\
\Pi_{22}^{n}=J_{n}^{\prime 2}\left(b_{\beta}\right), \quad \Pi_{33}^{n}=\frac{p_{\|}^{2}}{p_{\perp}^{2}} J_{n}^{2}\left(b_{\beta}\right),
\end{gathered}
$$

where

$$
\begin{array}{cc}
b_{\beta} \equiv \mu_{\beta} p_{\perp}, \quad \mu_{\beta} \equiv \frac{k}{m_{\beta} \Omega_{\beta}}, \quad D_{n \beta}=D_{n \beta}^{h}+i \frac{\nu_{\beta d}^{0}(p)}{\omega}, \\
D_{n \beta}^{h}=1-n Y_{\beta}, \quad Y_{\beta} \equiv \frac{\Omega_{\beta}}{\omega},
\end{array}
$$

and

$$
\begin{gathered}
\epsilon_{i j}^{N}=-\frac{4 \pi i n_{d 0}}{\omega} U_{i} S_{j}, \\
U_{i}=\frac{i}{\omega+i\left(\nu_{d}^{0}+\nu_{1}\right)} \sum_{\beta} \frac{q_{\beta}}{m_{\beta}^{2}} \sum_{n=-\infty}^{\infty} \int d^{3} p \frac{p_{\perp} f_{\beta 0} \sigma_{\beta}^{\prime}(p) p}{D_{n \beta}} R_{i 3}, \\
S_{j}=-\frac{1}{\omega^{2} n_{d 0}} \sum_{\beta} q_{\beta}^{2} \sum_{n=-\infty}^{\infty} \int d^{3} p \frac{p_{\perp}}{p} \nu_{\beta d}^{0}(p) \frac{d f_{\beta 0}}{d p} \frac{R_{j 3}}{D_{n \beta}},
\end{gathered}
$$

with

$$
R_{13}=\frac{n}{b_{\beta}} J_{n}^{2}\left(b_{\beta}\right), \quad R_{23}=-i J_{n}\left(b_{\beta}\right) J_{n}^{\prime}\left(b_{\beta}\right) .
$$


In these expressions we now use the explicit form of the Maxwellian distribution and retain only the contribution of the lowest harmonics, $n=0, \pm 1$. Making smallLarmor-radius expansions of the Bessel functions and their derivatives, mantaining terms up to second order, and expanding the denominator $D_{n \beta}^{h}+i \nu_{\beta d}^{0}(p) / \omega$ in powers of $i\left(\nu_{\beta d}^{0} / \omega\right) / D_{n \beta}^{h}$, retaining terms up to second order, we obtain for the nonvanishing components of the dielectric tensor the following expressions:

$$
\begin{gathered}
\tilde{\epsilon}_{11}^{h}=1-\frac{4 \pi}{3} \sum_{\beta} \frac{\eta_{\beta}}{1-Y_{\beta}^{2}}\left(\mathscr{I}_{0 \beta}^{1}-i E_{1 \beta} \mathscr{I}_{1 \beta}^{1}-E_{2 \beta} \mathscr{I}_{2 \beta}^{1}\right), \\
\tilde{\epsilon}_{12}^{h}=-i \frac{4 \pi}{3} \sum_{\beta} \frac{\eta_{\beta} Y_{\beta}}{1-Y_{\beta}^{2}}\left(\mathscr{I}_{0 \beta}^{2}-i E_{3 \beta} \mathscr{I}_{1 \beta}^{2}-E_{4 \beta} \mathscr{I}_{2 \beta}^{2}\right), \\
\tilde{\epsilon}_{22}^{h}=1-\frac{4 \pi}{3} \sum_{\beta} \eta_{\beta}\left[\frac{2}{5} \mu_{\beta}^{2}\left(I_{0 \beta}^{6}-i I_{1 \beta}^{6}-I_{2 \beta}^{6}\right)+\frac{1}{1-Y_{\beta}^{2}}\left(\mathscr{I}_{0 \beta}^{3}-i E_{1 \beta} \mathscr{I}_{1 \beta}^{3}-E_{2 \beta} \mathscr{I}_{2 \beta}^{3}\right)\right], \\
\tilde{\epsilon}_{33}^{h}=1-\frac{4 \pi}{3} \sum_{\beta} \eta_{\beta}\left[\mathscr{I}_{0 \beta}^{1}-i \mathscr{I}_{1 \beta}^{1}-\mathscr{I}_{2 \beta}^{1}+\frac{\mu_{\beta}^{2}}{5\left(1-Y_{\beta}^{2}\right)}\left(I_{0 \beta}^{6}-i E_{1 \beta} I_{1 \beta}^{6}-E_{2 \beta} I_{2 \beta}^{6}\right)\right], \\
\epsilon_{11}^{N}=-C\left[\sum_{\beta} N_{1 \beta}\left(\mathscr{J}_{0 \beta}^{3}-i E_{3 \beta} I_{1 \beta}^{3}\right)\right]\left[\sum_{\beta} N_{2 \beta}\left(I_{1 \beta}^{4}-i E_{3 \beta} I_{2 \beta}^{4}\right)\right], \\
\epsilon_{22}^{N}=C\left[\sum_{\beta} N_{1 \beta} Y_{\beta}\left(\mathscr{J}_{0 \beta}^{3}-i E_{5 \beta} I_{1 \beta}^{3}\right)\right] \\
\epsilon_{12}^{N}=i C\left[\sum_{\beta} N_{1 \beta} N_{2 \beta} Y_{\beta}\left(I_{1 \beta}^{4}-i I_{2 \beta}^{4}\right)\right], \\
{\left[\sum_{\beta} N_{1 \beta} Y_{\beta}\left(\mathscr{J}_{0 \beta}^{3}-i E_{5 \beta} I_{1 \beta}^{3}\right)\right]}
\end{gathered}
$$

where

$$
\begin{gathered}
\mathscr{I}_{n \beta}^{s} \equiv I_{n \beta}^{4}-\frac{1}{5} s \mu_{\beta}^{2} I_{n \beta}^{6}, \\
\mathscr{J}_{0 \beta}^{m} \equiv \int_{0}^{\infty} d p p^{m} H\left(p^{2}+C_{\beta}\right) f_{\beta 0} \\
I_{n \beta}^{m} \equiv \int_{0}^{\infty} d p p^{m} f_{\beta 0}\left(\frac{\nu_{\beta d}^{0}}{\omega}\right)^{n}
\end{gathered}
$$

with

$$
n=0,1,2, \quad s=1,2,3 .
$$

Explicit expressions for these integrals are given in Appendix B. Also in these 
expressions,

$$
\begin{gathered}
E_{1 \beta} \equiv \frac{1+Y_{\beta}^{2}}{1-Y_{\beta}^{2}}, \quad E_{2 \beta} \equiv \frac{1+3 Y_{\beta}^{2}}{\left(1-Y_{\beta}^{2}\right)^{2}}, \quad E_{3 \beta} \equiv \frac{2}{1-Y_{\beta}^{2}}, \\
E_{4 \beta} \equiv \frac{3+Y_{\beta}^{2}}{\left(1-Y_{\beta}^{2}\right)^{2}}, \quad E_{5 \beta} \equiv \frac{3-Y_{\beta}^{2}}{1-Y_{\beta}^{2}}, \quad C \equiv \frac{128 \pi^{4} a}{9 \omega^{2}\left[\omega+i\left(\nu_{d}^{0}+\nu_{1}\right)\right]}, \\
\eta_{\beta} \equiv \frac{X_{\beta}}{n_{\beta 0} m_{\beta} T_{\beta}}, \quad N_{1 \beta} \equiv \frac{q_{\beta}^{2} \mu_{\beta} Y_{\beta}}{m_{\beta}\left(1-Y_{\beta}^{2}\right)}, \quad N_{2 \beta} \equiv \frac{N_{1 \beta}}{T_{\beta}} .
\end{gathered}
$$

In this approximation, $\nu_{d}^{0}+\nu_{1}$ is given by

$$
\nu_{d}^{0}+\nu_{1}=\frac{4 \pi^{2} a}{3} \sum_{\beta} q_{\beta}^{2}\left[6\left(\mathscr{J}_{0 \beta}^{1}-i I_{1 \beta}^{1}\right)-i 2 \mu_{\beta}^{2} \frac{Y_{\beta}^{2}}{1-Y_{\beta}^{2}} I_{1 \beta}^{3}\right] .
$$

\section{The magnetosonic wave}

The dispersion relation for the magnetosonic wave can be written formally as

$$
\Lambda=N^{2}-\frac{\epsilon_{x x} \epsilon_{y y}-\epsilon_{x y} \epsilon_{y x}}{\epsilon_{x x}}=0 .
$$

In order to study the simplest effect produced by the inclusion of dust particles with variable charge in the propagation properties of this wave, we make the following approximations in the calculation of the $\epsilon_{i j}$, which are given in Sec. 4: we neglect contributions arising from terms that contain $\left(\nu_{\beta d}^{0} / \omega\right)^{2}$; we neglect terms in $\mu_{\beta}^{2}$ and higher orders in $\mu_{\beta}^{2}$, which corresponds to making a small-Larmor-radius approximation (in this case a cold-plasma approximation); and we work in a frequency regime such that $Y_{\beta}^{2} \gg 1$, which is the appropriate regime for magnetosonic waves. In this case $\epsilon_{i j} \simeq \tilde{\epsilon}_{i j}^{h}$, and the dispersion relation can be cast in the form

$$
\Lambda=N^{2}-\frac{\epsilon_{x x}^{2}+\epsilon_{x y}^{2}}{\epsilon_{x x}}=0,
$$

with

where

$$
\begin{gathered}
\epsilon_{x x}=1+\chi_{x x}^{(0)}+i \chi_{x x}^{(1)}, \\
\epsilon_{x y}=i \frac{\mathscr{C}}{\omega},
\end{gathered}
$$

$$
\begin{gathered}
\chi_{x x}^{(n)}=\frac{4 \pi}{3} \sum_{\beta} \frac{c^{2}}{V_{A \beta}^{2}} \frac{I_{n \beta}^{4}}{n_{\beta 0} m_{\beta} T_{\beta}}, \quad n=0,1, \\
\mathscr{C}=\frac{4 \pi}{3} \omega \sum_{\beta} \frac{c^{2}}{V_{A \beta}^{2}} \frac{Y_{\beta}}{n_{\beta 0} m_{\beta} T_{\beta}} I_{0 \beta}^{4},
\end{gathered}
$$

and

$$
V_{A \beta}^{2} \equiv \frac{B_{0}^{2}}{4 \pi n_{\beta 0} m_{\beta}}
$$

is the square of the Alfvén velocity of particles of species $\beta$. 
Using the explicit expression for $I_{0 \beta}^{4}$ and remembering the approximations utilized, we can write

$$
\mathscr{C}=\frac{4 \pi c}{B_{0}} \sum_{\beta} n_{\beta 0} q_{\beta},
$$

which, using the quasineutrality condition, can be cast in the form

$$
\mathscr{C}=\frac{4 \pi c}{B_{0}} Z_{d} e n_{d 0} .
$$

We observe that in the absence of dust particles $\mathscr{C}=0$, which implies that $\epsilon_{x y}=0$, and the dispersion relation reduces to $N^{2}=\epsilon_{x x}=1+c^{2} / V_{A i}^{2}$, which is the traditional form of this relation for magnetosonic waves in the cold-plasma approximation (Krall and Trivelpiece 1973). We note that even if the dust particles have fixed charges, $\mathscr{C} \neq 0$ which implies $\epsilon_{x y} \neq 0$, and produces modification in the dispersion relation.

We can write

$$
\chi_{x x}^{(0)} \simeq \frac{c^{2}}{V_{A i}^{2}},
$$

where we have used the condition that $V_{A i}^{2} \ll V_{A e}^{2}$, and

$$
\chi_{x x}^{(1)}=\frac{4}{3}(2 \pi)^{1 / 2} \frac{a^{2} n_{d 0}}{\omega} \sum_{\beta} \frac{c^{2}}{V_{A \beta}^{2}} v_{T \beta}\left[\left(2+\chi_{i}\right) \delta_{\beta i}+e^{-\xi^{2}}\left(\xi^{2}+2\right) \delta_{\beta e}\right] \equiv \frac{\mathscr{B}}{\omega} .
$$

The relevant components of the dielectric tensor that occur in the dispersion relation can be written as

$$
\epsilon_{x x}=\mathscr{A}+i \frac{\mathscr{B}}{\omega}, \quad \epsilon_{x y}=i \frac{\mathscr{C}}{\omega},
$$

where $\mathscr{A} \equiv 1+\chi_{x x}^{(0)}$, and $\mathscr{A}, \mathscr{B}$ and $\mathscr{C}$ are positive quantities. The real and imaginary parts of $\Lambda$ are

where

$$
\begin{gathered}
\Lambda_{r}=\frac{c^{2} k^{2}}{\omega^{2}}-\mathscr{A}[1-F(\omega)], \\
\Lambda_{i}=-\frac{\mathscr{B}}{\omega}[1+F(\omega)],
\end{gathered}
$$

$$
\begin{gathered}
F(\omega) \equiv \frac{\alpha_{2}{ }^{2}}{\omega^{2}+\alpha_{1}{ }^{2}}, \\
\alpha_{1} \equiv \frac{\mathscr{B}}{\mathscr{A}}, \quad \alpha_{2} \equiv \frac{\mathscr{C}}{\mathscr{A}} .
\end{gathered}
$$

From $\Lambda_{r}\left(w_{r}\right)=0$, we get the real part of the propagation frequency

$$
\begin{gathered}
\omega_{r}^{2}=\frac{1}{2}\left[-Q+\left(Q^{2}+4 \omega_{0}^{2} \alpha_{1}^{2}\right)^{1 / 2}\right], \\
Q \equiv \alpha_{1}^{2}-\left(\alpha_{2}^{2}+\omega_{0}^{2}\right), \\
\omega_{0}^{2} \equiv \frac{c^{2} k^{2}}{\mathscr{A}}=\frac{k^{2} V_{A i}^{2}}{1+V_{A i}^{2} / c^{2}},
\end{gathered}
$$

which in the limit $n_{d 0} \rightarrow 0$ gives $\omega_{r}^{2}=\omega_{0}^{2}$, the usual result for the frequency of the magnetosonic wave. 


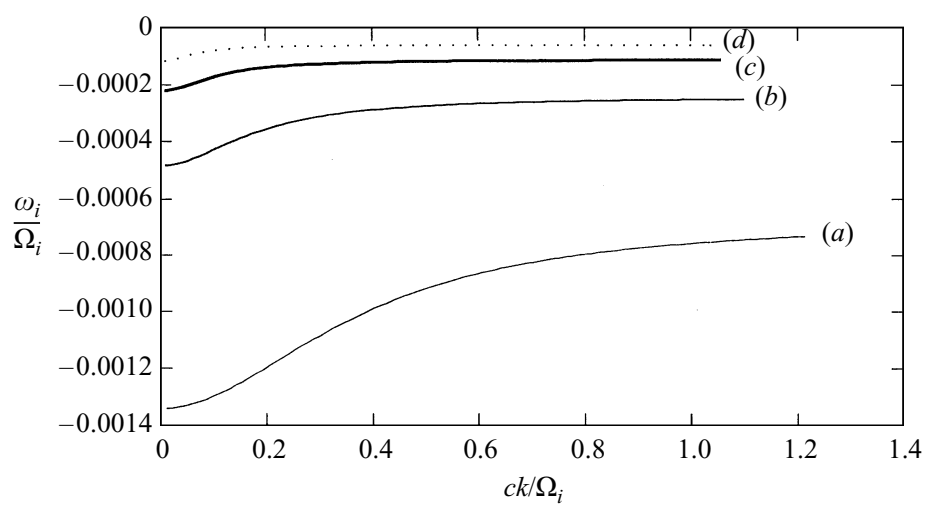

Figure 1. $\omega_{i} / \Omega_{i}$ as a function of $c k / \Omega_{i}$ for $T=1.5 \times 10^{4} \mathrm{eV}, n_{d 0}=10^{4} \mathrm{~cm}^{-3}, n_{i 0}=10^{8} \mathrm{~cm}^{-3}$, $a=10^{-4} \mathrm{~cm}$, and several values of the external magnetic field $B_{0}:(a) 0.2 \mathrm{~T} ;(b) 0.3 \mathrm{~T} ;(c) 0.4$ $\mathrm{T} ;(d) 0.5 \mathrm{~T}$.

The imaginary part of the frequency is obtained from

$$
\omega_{i}=-\frac{\Lambda_{i}\left(k, \omega_{r}\right)}{\left.(\partial / \partial \omega) \Lambda_{r}(k, \omega)\right|_{\omega=\omega_{r}}},
$$

and is given in this case by

$$
\omega_{i}=-\frac{\omega_{r}^{2}}{2} \alpha_{1} \frac{1+F\left(\omega_{r}\right)}{\omega_{0}^{2}+F\left(\omega_{r}\right) G\left(\omega_{r}\right)},
$$

where

$$
G\left(\omega_{r}\right) \equiv \frac{\omega_{r}^{4}}{\omega_{r}^{2}+\alpha_{1}^{2}}
$$

We note that $\omega_{i}=0$ if $n_{d 0}=0$, and when $n_{d 0} \neq 0$ we have $w_{i}<0$, showing that the wave is absorbed. This absorption comes from the presence of the dust particles with variable charges in the plasma.

We note also that if we do not have charge variation, $\mathscr{C} \neq 0$ but $\mathscr{B}=0$, because it originates from terms that are dependent on $\nu_{\beta d}^{0}=0$. This implies that $\omega_{r}$ will be modified, but we shall still have $\omega_{i}=0$.

In what follows we make several numerical estimates of the relevant parameters that describe the propagation and absorption of the magnetosonic wave in the proposed model. All the results presented here were calculated using $a=10^{-4} \mathrm{~cm}$ for the radius of the dust grain. We have checked, for each set of parameters employed, if the limits $a \ll \lambda_{D}$ and $a \ll \rho$, which restrict the validity of the model, are satisfied. All the figures presented are consistent with these limitations. The equilibrium densities of electrons, ions and dust, and the equilibrium charge of the dust particles, are obtained given two of these quantities and determining the other two in such a way that $(2.15)$ and $(2.20)$, which characterize the equilibrium, are satisfied.

In Fig. 1 we show $\omega_{i} / \Omega_{i}$ as a function of $c k / \Omega_{i}$, for several values of the external magnetic field $B_{0}$. The curves labelled by $(a),(b),(c)$ and $(d)$ correspond to $B_{0}=$ $0.2,0.3,0.4$ and $0.5 \mathrm{~T}$ respectively. The fixed parameters are $T=1.5 \times 10^{4} \mathrm{eV}$, $n_{d 0}=10^{4} \mathrm{~cm}^{-3}, n_{i 0}=10^{8} \mathrm{~cm}^{-3}$ and $a=10^{-4} \mathrm{~cm}$, which give $Z_{d} \simeq 9766$. We see that the absolute value of $\omega_{i} / \Omega_{i}$ decreases with increasing $B_{0}$, and for $B_{0}>0.6 \mathrm{~T}$ 


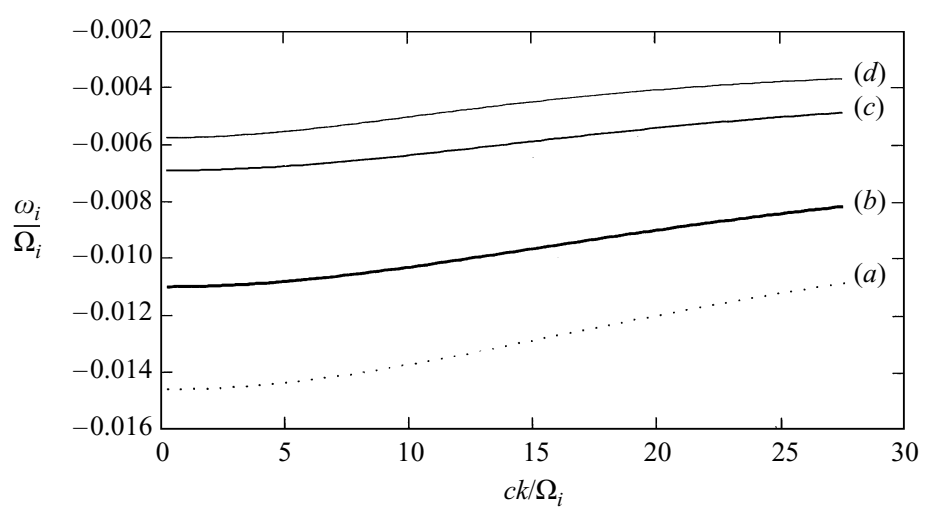

Figure 2. $\omega_{i} / \Omega_{i}$ as a function of $c k / \Omega_{i}$ for $B_{0}=5 \times 10^{-3} \mathrm{~T}$ and several values of the temperature $T$ of electrons and ions: $(a) 100 \mathrm{eV} ;(b) 50 \mathrm{eV} ;(c) 10 \mathrm{eV} ;(d) 5 \mathrm{eV}$. The other parameters are as in Fig. 1.

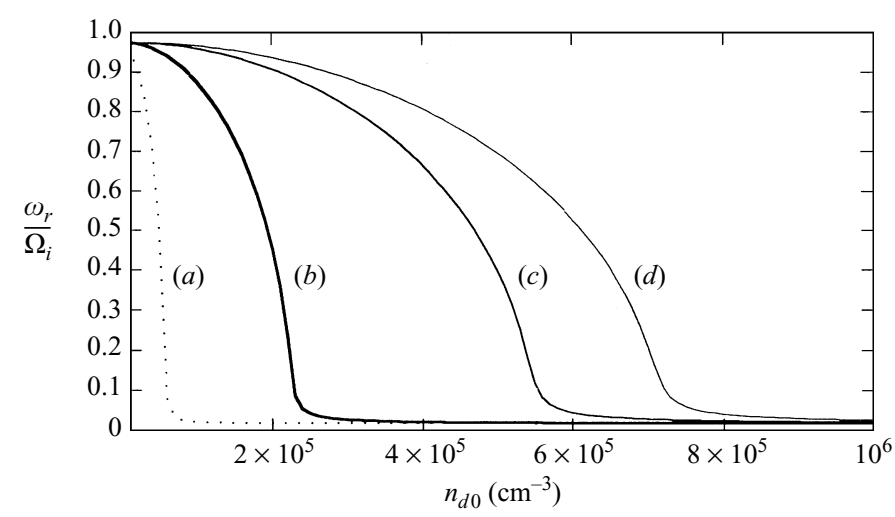

Figure 3. $\omega_{r} / \Omega_{i}$ as a function of $n_{d 0}$ for $a=10^{-4} \mathrm{~cm}, B_{0}=5 \times 10^{-3} \mathrm{~T}, n_{i 0}=10^{8} \mathrm{~cm}^{-3}$, $c k / \Omega_{i}=0.5$ and several values of the temperature $T$ of electrons and ions: (a) $15 \mathrm{keV}$; (b) $1 \mathrm{keV}$; (c) $170 \mathrm{eV}$; (d) $100 \mathrm{eV}$.

the curves become nearly horizontal approaching the axis for larger values of $B_{0}$. We remark that increasing $B_{0}$ causes the ions and electrons to be more closely bound to the magnetic field lines and therefore to contribute less to the variation of the currents that charge the dust particles.

In Fig. 2 we show $\omega_{i} / \Omega_{i}$ as a function of $c k / \Omega_{i}$, for several values of the temperature $T$ of electrons and ions. The curves labelled by $(a),(b),(c)$ and $(d)$ correspond to $T=100,50,10$ and $5 \mathrm{eV}$ respectively. The external magnetic field is $B_{0}=5 \times 10^{-3}$ $\mathrm{T}$, and the other parameters are the same as used in Fig. 1. In this case $Z_{d}$ is approximately $6164,8302,9606$ and 9694 , for curves $(a),(b),(c)$ and $(d)$ respectively. We see that the increase of the absolute value of $\omega_{i} / \Omega_{i}$ increases with increasing temperature of electrons and ions; for very low values of the temperature we obtain horizontal lines, and the absolute value of $\omega_{i} / \Omega_{i}$ approaches to zero. We remark that with increasing temperature, electrons and ions contribute more to the variation of the currents that charge the dust particles.

Figure 3 shows $\omega_{r} / \Omega_{i}$ as a function of $n_{d 0}$, for several values of the temperature $T$ of electrons and ions. The curves labelled by $(a),(b),(c)$ and $(d)$ correspond to 


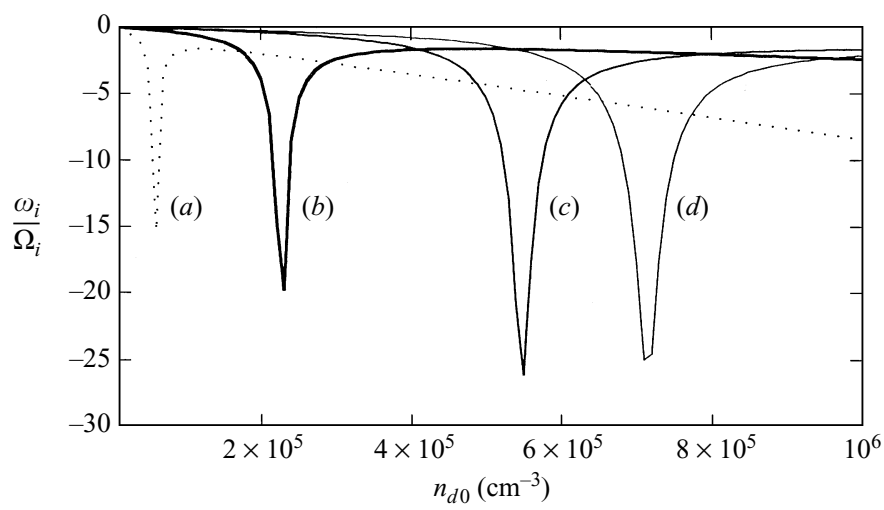

Figure 4. $\omega_{i} / \Omega_{i}$ as a function of $n_{d 0}$ for several values of the temperature $T$ of electrons and ions. The other parameters are as in Fig. 3.

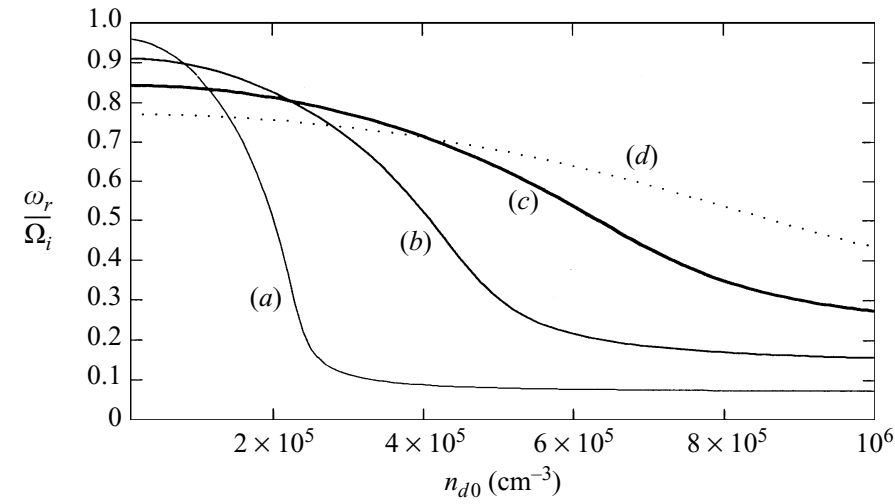

Figure 5. $\omega_{r} / \Omega_{i}$ as a function of $n_{d 0}$ for $a=10^{-4} \mathrm{~cm}, T=1.5 \times 10^{4} \mathrm{eV}, n_{i 0}=10^{8} \mathrm{~cm}^{-3}$ $c k / \Omega_{i}=0.5$ and several values of the external magnetic field $B_{0}:(a) 2 \times 10^{-2} \mathrm{~T}$; (b) $4 \times 10^{-2} \mathrm{~T}$; (c) $6 \times 10^{-2} \mathrm{~T} ;(d) 8 \times 10^{-2} \mathrm{~T}$.

$T=15 \mathrm{keV}, 1 \mathrm{keV}, 170 \mathrm{eV}$ and $100 \mathrm{eV}$ respectively. The fixed parameters are $a=10^{-4} \mathrm{~cm}, B_{0}=5 \times 10^{-3} \mathrm{~T}, n_{i 0}=10^{8} \mathrm{~cm}^{-3}$ and $c k / \Omega_{i}=0.5$. We see that $\omega_{r} / \Omega_{i}$ decays sharply to small values with increasing $n_{d 0}$, and that this depends on the temperature of electrons and ions: the critical value of $n_{d 0}$ where the abrupt decay occurs diminishes with increasing temperature.

In Fig. 4 we show $\omega_{i} / \Omega_{i}$ as a function of $n_{d 0}$, for the same parameters as used in Fig. 3 and the same values of electron and ion temperature. We remark that at the values of $n_{d 0}$ where $\omega_{r} / \Omega_{i}$ decays to small values, $\omega_{i} / \Omega_{i}$ presents minimum points. These minimum points are shifted to higher values of $n_{d 0}$ with decreasing temperature.

Figures 5 and 6 show, for $a=10^{-4} \mathrm{~cm}, T=1.5 \times 10^{4} \mathrm{eV}, n_{i 0}=10^{8} \mathrm{~cm}^{-3}$ and $c k / \Omega_{i}=0.5, \omega_{r} / \Omega_{i}$ and $\omega_{i} / \Omega_{i}$ as functions of $n_{d 0}$, for several values of the external magnetic field $B_{0}:(a) 2 \times 10^{-2} \mathrm{~T},(b) 4 \times 10^{-2} \mathrm{~T},(c) 6 \times 10^{-2} \mathrm{~T}$ and $(d) 8 \times 10^{-2} \mathrm{~T}$. We see the same kind of behaviour in these figures that we have observed in Figs 3 and 4 . 


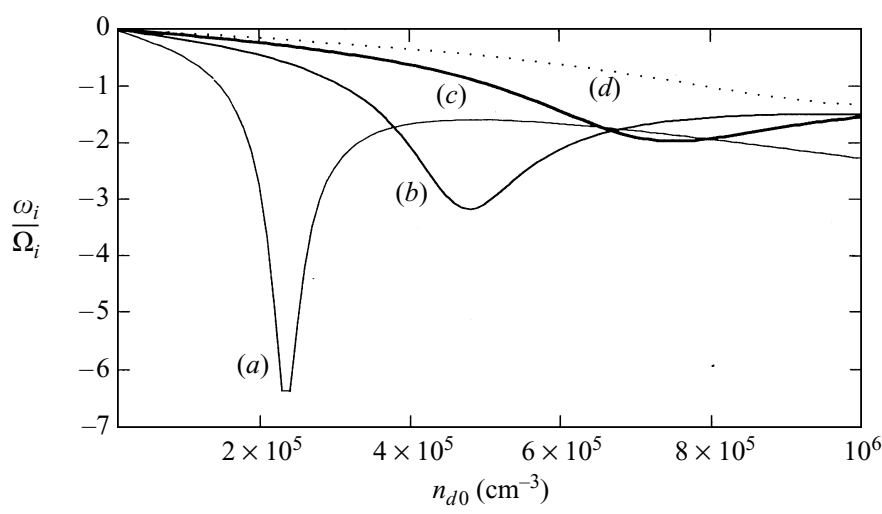

Figure 6. $\omega_{i} / \Omega_{i}$ as a function of $n_{d 0}$ for several values of the external magnetic field. The other parameters are as in Fig. 5 .

We remark that variations in the temperature of electrons and ions and in the external magnetic field have the same type of effect on the enhancement of the currents that charge the dust particles: these currents grow with increasing temperature or decreasing magnetic field.

\section{Summary and conclusions}

In this paper we have developed a complete treatment, in the framework of kinetic theory, in order to obtain the dielectric tensor that describes a plasma composed of electrons, ions and dust particles immersed in an external magnetic field, taking the charge variation of the dust particles explicitly into account.

The general expressions obtained show that the dielectric response of the plasma is modified in two ways by the charge variation of the dust particles. First, the denominator that usually occurs in the expression for the components of the dielectric tensor of a homogeneous plasma in the presence of an external magnetic field is modified by the addition of a purely imaginary term. This term can give rise to amplification or absorption of the wave that is being considered. Also, in some situations, we can have competition between Landau damping and the damping due to the presence of the dust particles with variable charge. Secondly, due to the charge variation of the dust particles, the dielectric tensor components have an additional term that depends strongly on the process of charging of the dust particles.

We have presented general expressions for the dielectric tensor components, and have also derived particular expressions for the tensor, suitable for perpendicular propagation and Maxwellian distribution functions of electrons and ions.

We have made a preliminary application of this formalism to the magnetosonic wave. The magnetosonic wave propagates perpendicularly to an external magnetic field, and is usually an undamped mode. We have shown that the inclusion of dust particles, using the model proposed in this paper in its simplest form, leads to the possibility of the magnetosonic wave being absorbed. We intend to explore this application more deeply, retaining the effects of charge variation of the dust particles that are included in $\epsilon_{i j}^{N}$. 


\section{Appendix A}

The quantities $R_{i j}$ are given by

$$
\begin{aligned}
& R_{x x}^{R}=J_{n}^{\prime 2}+\cos ^{2} \psi\left(\frac{n^{2}}{b_{\beta}^{2}} J_{n}^{2}-J_{n}^{\prime 2}\right), \quad R_{x x}^{I}=0, \\
& R_{x y}^{R}=\left(\frac{n^{2}}{b_{\beta}^{2}} J_{n}^{2}-J_{n}^{\prime 2}\right) \sin \psi \cos \psi, \quad R_{x y}^{I}=\frac{n}{b_{\beta}} J_{n} J_{n}^{\prime}, \\
& R_{x z}^{R}=\frac{n}{b_{\beta}} J_{n}^{2} \cos \psi, \quad R_{x z}^{I}=J_{n} J_{n}^{\prime} \sin \psi, \\
& R_{y y}^{R}=\frac{n^{2}}{b_{\beta}^{2}} J_{n}^{2}+\cos ^{2} \psi\left(J_{n}^{\prime 2}-\frac{n^{2}}{b_{\beta}^{2}} J_{n}^{2}\right), \quad R_{y y}^{I}=0, \\
& R_{y z}^{R}=\frac{n}{b_{\beta}} J_{n}^{2} \sin \psi, \quad R_{y z}^{I}=-J_{n} J_{n}^{\prime} \cos \psi, \\
& R_{z z}^{R}=J_{n}^{2}, \quad R_{z z}^{I}=0,
\end{aligned}
$$

with $R_{i j}^{R}=R_{j i}^{R}$ and $R_{i j}^{I}=-R_{j i}^{I}$.

The quantities $R_{i j}$ depend on particle species, but are written without explicit reference to the $\beta$ index in order to not burden the notation.

In the above expressions the Bessel functions $J_{n}$ and their derivatives depend on the argument $b_{\beta}=k_{\perp} p_{\perp} / m_{\beta} \boldsymbol{\Omega}_{\beta}$, and $\psi=\tan ^{-1}\left(k_{y} / k_{x}\right)$ is the angle between the vector $\mathbf{k}_{\perp}$ and the $x$ axis; $k_{\perp}$ is the perpendicular component of the wave vector. In the situation treated in this paper, $\psi=0$.

\section{Appendix B}

We give the integrals for the computation of the dielectric tensor for dusty plasmas when $k_{\|}=0$ and $f_{\beta 0}$ is a Maxwellian distribution function.

Expressions for $I_{0 \beta}^{m}$

(a) For even $m$,

(b) for odd $m$,

$$
\begin{gathered}
I_{0 \beta}^{2 l}=\frac{n_{\beta 0}}{\pi} \frac{(2 l-1) ! !}{2^{l+1}}\left(2 m_{\beta} T_{\beta}\right)^{l-1}, \\
I_{0 \beta}^{4}=\frac{3}{4 \pi} n_{\beta 0} m_{\beta} T_{\beta}, \quad I_{0 \beta}^{6}=\frac{15}{4 \pi} n_{\beta 0}\left(m_{\beta} T_{\beta}\right)^{2} .
\end{gathered}
$$

$$
I_{0 \beta}^{2 l+1}=\frac{n_{\beta 0} l !}{2 \pi^{3 / 2}}\left(2 m_{\beta} T_{\beta}\right)^{l-1 / 2} .
$$

Expressions for $I_{1 \beta}^{m}$

$$
\begin{aligned}
& I_{1 \beta}^{m}=\frac{\pi a^{2} n_{d 0}}{\omega m_{\beta}}\left(\mathscr{J}_{0 \beta}^{m+1}+C_{\beta} \mathscr{J}_{0 \beta}^{m-1}\right), \\
& I_{1 \beta}^{1}=\frac{\pi a^{2} n_{d 0}}{\omega m_{\beta}}\left(\mathscr{J}_{0 \beta}^{2}+C_{\beta} \mathscr{J}_{0 \beta}^{0}\right), \quad I_{1 \beta}^{3}=\frac{\pi a^{2} n_{d 0}}{\omega m_{\beta}}\left(\mathscr{J}_{0 \beta}^{4}+C_{\beta} \mathscr{J}_{0 \beta}^{2}\right), \\
& I_{1 \beta}^{4}=\frac{\pi a^{2} n_{d 0}}{\omega m_{\beta}}\left(\mathscr{J}_{0 \beta}^{5}+C_{\beta} \mathscr{J}_{0 \beta}^{3}\right), \quad I_{1 \beta}^{6}=\frac{\pi a^{2} n_{d 0}}{\omega m_{\beta}}\left(\mathscr{J}_{0 \beta}^{7}+C_{\beta} \mathscr{J}_{0 \beta}^{5}\right) .
\end{aligned}
$$


Expressions for $I_{2 \beta}^{m}$

$$
\begin{gathered}
I_{2 \beta}^{m}=\left(\frac{\pi a^{2} n_{d 0}}{\omega m_{\beta}}\right)^{2}\left(\mathscr{J}_{0 \beta}^{m+2}+2 C_{\beta} \mathscr{J}_{0 \beta}^{m}+C_{\beta}^{2} \mathscr{J}_{0 \beta}^{m-2}\right), \\
I_{2 \beta}^{4}=\left(\frac{\pi a^{2} n_{d 0}}{\omega m_{\beta}}\right)^{2}\left(\mathscr{J}_{0 \beta}^{6}+2 C_{\beta} \mathscr{J}_{0 \beta}^{4}+C_{\beta}^{2} \mathscr{J}_{0 \beta}^{2}\right), \\
I_{2 \beta}^{6}=\left(\frac{\pi a^{2} n_{d 0}}{\omega m_{\beta}}\right)^{2}\left(\mathscr{J}_{0 \beta}^{8}+2 C_{\beta} \mathscr{J}_{0 \beta}^{6}+C_{\beta}^{2} \mathscr{J}_{0 \beta}^{4}\right) .
\end{gathered}
$$

Expressions for $\mathscr{J}_{0 \beta}^{m}$

(a) For $m=0$,

$$
\mathscr{J}_{0 \beta}^{0}=\frac{n_{\beta 0}}{2 \pi\left(2 m_{\beta} T_{\beta}\right)}\left[1-\delta_{\beta e} \operatorname{erf}(\xi)\right] .
$$

(b) For even $m$,

$$
\begin{aligned}
& \mathscr{J}_{0 \beta}^{2 l}=\frac{(2 l) !}{l ! 2^{2 l-1}}\left\{\frac{n_{\beta 0}}{4 \pi}\left(2 m_{\beta} T_{\beta}\right)^{l-1}-\frac{\delta_{\beta e} n_{e 0}}{\pi^{3 / 2}}\left(2 m_{e} T_{e}\right)^{l-1}\left[-\frac{e^{-\xi^{2}}}{2} \sum_{k=1}^{l} \frac{(2 \xi)^{2 k-1} k !}{(2 k) !}\right.\right. \\
& \left.\left.+\frac{\pi^{1 / 2}}{4} \operatorname{erf}(\xi)\right]\right\} \\
& \mathscr{J}_{0 \beta}^{2}=\left\{\frac{n_{\beta 0}}{4 \pi}-\frac{\delta_{\beta e} n_{e 0}}{\pi^{3 / 2}}\left[-\frac{e^{-\xi^{2}}}{2} \xi+\frac{\pi^{1 / 2}}{4} \operatorname{erf}(\xi)\right]\right\}, \\
& \mathscr{J}_{0 \beta}^{4}=\frac{3}{2}\left\{\frac{n_{\beta 0}}{4 \pi}\left(2 m_{\beta} T_{\beta}\right)-\frac{\delta_{\beta e} n_{e 0}}{\pi^{3 / 2}}\left(2 m_{e} T_{e}\right)\left[-\frac{e^{-\xi^{2}}}{2}\left(\frac{2 \xi^{3}}{3}+\xi\right)+\frac{\pi^{1 / 2}}{4} \operatorname{erf}(\xi)\right]\right\}, \\
& \mathscr{J}_{0 \beta}^{6}=\frac{15}{4}\left\{\frac{n_{\beta 0}}{4 \pi}\left(2 m_{\beta} T_{\beta}\right)^{2}-\frac{\delta_{\beta e} n_{e 0}}{\pi^{3 / 2}}\left(2 m_{e} T_{e}\right)^{2}\left[-\frac{e^{-\xi^{2}}}{2}\left(\frac{4 \xi^{5}}{15}+\frac{2 \xi^{3}}{3}+\xi\right)\right.\right. \\
& \left.\left.+\frac{\pi^{1 / 2}}{4} \operatorname{erf}(\xi)\right]\right\} \\
& \mathscr{J}_{0 \beta}^{8}=\frac{105}{8}\left\{\frac{n_{\beta 0}}{4 \pi}\left(2 m_{\beta} T_{\beta}\right)^{3}-\frac{\delta_{\beta e n_{e 0}}}{\pi^{3 / 2}}\left(2 m_{e} T_{e}\right)^{3}\left[-\frac{e^{-\xi^{2}}}{2}\left(\frac{8 \xi^{7}}{105}+\frac{4 \xi^{5}}{15}+\frac{2 \xi^{3}}{3}+\xi\right)\right.\right. \\
& \left.\left.+\frac{\pi^{1 / 2}}{4} \operatorname{erf}(\xi)\right]\right\} \text {. }
\end{aligned}
$$

(c) For odd, $m$

$$
\begin{gathered}
\mathscr{J}_{0 \beta}^{2 l+1}=\frac{l ! n_{\beta 0}\left(2 m_{\beta} T_{\beta}\right)^{(2 l-1) / 2}}{2 \pi^{3 / 2}}\left(\delta_{\beta i}+\delta_{\beta e} e^{-\xi^{2}} \sum_{k=0}^{l} \frac{\xi^{2 l}}{k !}\right), \\
\mathscr{J}_{0 \beta}^{1}=\frac{n_{\beta 0}\left(2 m_{\beta} T_{\beta}\right)^{-1 / 2}}{2 \pi^{3 / 2}}\left(\delta_{\beta i}+\delta_{\beta e} e^{-\xi^{2}}\right), \\
\mathscr{J}_{0 \beta}^{3}=\frac{n_{\beta 0}\left(2 m_{\beta} T_{\beta}\right)^{1 / 2}}{2 \pi^{3 / 2}}\left[\delta_{\beta i}+\delta_{\beta e} e^{-\xi^{2}}\left(\xi^{2}+1\right)\right],
\end{gathered}
$$




$$
\begin{gathered}
\mathscr{J}_{0 \beta}^{5}=\frac{2 n_{\beta 0}\left(2 m_{\beta} T_{\beta}\right)^{3 / 2}}{2 \pi^{3 / 2}}\left[\delta_{\beta i}+\delta_{\beta e} e^{-\xi^{2}}\left(\frac{\xi^{4}}{2}+\xi^{2}+1\right)\right], \\
\mathscr{J}_{0 \beta}^{7}=\frac{6 n_{\beta 0}\left(2 m_{\beta} T_{\beta}\right)^{5 / 2}}{2 \pi^{3 / 2}}\left[\delta_{\beta i}+\delta_{\beta e} e^{-\xi^{2}}\left(\frac{\xi^{6}}{6}+\frac{\xi^{4}}{2}+\xi^{2}+1\right)\right],
\end{gathered}
$$

where $\xi^{2} \equiv\left|\chi_{e}\right|$

\section{References}

Caldela, R. A., Schneider, R. S. and Ziebell, L. F. 1989 The dispersion relation and the dielectric tensor of inhomogeneous magnetized plasmas. J. Plasma Phys. 42, 165.

Chang, J. S. and Spariosu, K. 1993 Dust particle charging characteristics under a collisionless magneto-plasma. J. Phys. Soc. Japan 62, 97.

D'Angelis, U. 1992 The physics of dusty plasmas. Physica Scripta 45, 465.

Draine, B. T. and Salpeter, E. E. 1979 On the physics of dust grains in hot gas. Astrophys. J. $231,77$.

Hutchinson, I. H. 1987 Principles of Plasma Diagnostics. Cambridge University Press.

Krall, N. A. and Trivelpiece, A. W. 1973 Principles of Plasma Physics. McGraw-Hill, New York (reprinted 1986 San Francisco Press).

Melandsø, F., Aslaksen, T. K. and Havnes, O. 1993 A kinetic model for dust acoustic waves applied to planetary rings. J. of Geophys. Res. 98, 13315.

Rao, N. N. 1995 Magnetoacoustic modes in a magnetized dusty plasma. J. Plasma Phys. 53, 317.

Rao, N. N., Shukla, P. K. and Yu, M. Y. 1990 Dust-acoustic waves in dusty plasmas. Planet. Space Sci. 38, 543.

Shukla, P. K. 1992 Low-frequency modes in dusty plasmas. Physica Scripta 45, 504.

Spitzer, L. 1978 Physical Processes in the Interstellar Medium. Wiley, New York.

Tsytovich, V. N. and Havnes, O. 1993 Charging processes, dispersion properties and anomalous transport in dusty plasma. Comments Plasma Phys. Contr. Fusion 15, 267.

Varma, R. K., Shukla, P. K. and Krishan, V. 1993 Electrostatic oscillations in the presence of grain-charge perturbations in dusty plasmas. Phys. Rev. E47, 3612.

Vladimirov, S. V. 1994 Propagation of waves in dusty plasmas with variable charges on dust particles. Phys. Plasmas 1, 2762. 